\title{
Search strategy is regulated by somatostatin signaling and deep brain photoreceptors in zebrafish
}

\author{
Eric J. Horstick*, Yared Bayleyen, Jennifer L. Sinclair and Harold A. Burgess ${ }^{*}$
}

\begin{abstract}
Background: Animals use sensory cues to efficiently locate resources, but when sensory information is insufficient, they may rely on internally coded search strategies. Despite the importance of search behavior, there is limited understanding of the underlying neural mechanisms in vertebrates.

Results: Here, we report that loss of illumination initiates sophisticated light-search behavior in larval zebrafish. Using three-dimensional tracking, we show that at the onset of darkness larvae swim in a helical trajectory that is spatially restricted in the horizontal plane, before gradually transitioning to an outward movement profile. Local and outward swim patterns display characteristic features of area-restricted and roaming search strategies, differentially enhancing phototaxis to nearby and remote sources of light. Retinal signaling is only required to initiate arearestricted search, implying that photoreceptors within the brain drive the transition to the roaming search state. Supporting this, orthopediaA mutant larvae manifest impaired transition to roaming search, a phenotype which is recapitulated by loss of the non-visual opsin opn4a and somatostatin signaling.
\end{abstract}

Conclusion: These findings define distinct neuronal pathways for area-restricted and roaming search behaviors and clarify how internal drives promote goal-directed activity.

Keywords: Zebrafish, Search, Motivation, Goal-directed behavior, Non-visual photoreceptor, CRISPR, Orthopedia, Somatostatin, sst1.1, Melanopsin, opn4a

\section{Background}

Essential resources tend to be clustered, rather than uniformly distributed in the natural environment. Accordingly, animals use a range of behavioral strategies to search for resource rich areas [1]. Search behaviors have been characterized in invertebrate species [2-4], including in C. elegans, where detailed underlying neuronal circuits have been described [5-7]. Although sophisticated search behaviors under natural conditions have also been catalogued in vertebrates, surprisingly few studies have exploited vertebrate models to probe genetic factors and neuronal connections that initiate and maintain active search states [8-11].

To date, the powerful repertoire of neurogenetic tools available in zebrafish have been primarily applied to

\footnotetext{
*Correspondence: eric.horstick@nih.gov; burgessha@mail.nih.gov Division of Developmental Biology, Eunice Kennedy Shriver National Institute of Child Health and Human Development, Bethesda, MD 20892, USA
}

decoding neural mechanisms for acute behavioral responses to sensory stimuli $[12,13]$. For instance, larval zebrafish show phototaxis toward restricted areas of illumination in dark environments [14, 15], maneuver within thermal and chemical gradients [16, 17], and actively oppose water currents by swimming against whole-field visual motion. Thus, larvae use sensory information in several modalities to actively navigate within the environment, initiating approach or avoidance behaviors as required. However, much less is known about whether larvae efficiently locate resources that are not in the immediate vicinity. Search behavior requires the maintenance of an internal state that appropriately regulates motor activity, and potentially also modulates sensory thresholds to facilitate the discovery of desirable resources $[18,19]$. Short-term internal states such as arousal and hunger, and movement profiles consistent with exploratory behavior are present in larval stage 
zebrafish [10, 11, 20-22], raising the possibility that autogenic search behavior has also developed.

One promising avenue to investigate search behavior is the hyperactive response of zebrafish larvae to a complete loss of illumination [23-25]. At the onset of darkness, zebrafish larvae become hyperactive for 5-10 min, before settling into a low-activity sleep-like state. The hyperactive state is referred to as the visual motor response, or dark photokinesis [26]. Hyperactivity propels larvae out of dark regions - slower movement in the light causes larvae to aggregate in illuminated regions, an example of classical photokinesis behavior [26, 27].

Here, we investigated whether the locomotor hyperactivity that occurs during dark photokinesis is randomly directed or rather shows spatial patterning that might facilitate light-search behavior. We found that larval swim trajectories during the first few minutes of photokinesis possess characteristic features of a widely utilized animal foraging strategy known as area-restricted search $[2,3,8,28]$. Area-restricted search is a strategy for temporarily restricting movement to the region where a specific resource was last detected. Typically, area-restricted search is a transient behavior that transitions to a roaming search strategy if the resource is not quickly located [1]. Similarly, we show that after 2-3 min of locally restricted movement, larval swim paths transition to an outward trajectory, consistent with a roaming strategy for light sources that are outside the initial visual range. We demonstrate that the distinct swimming patterns associated with area-restricted and roaming search states respectively increase the ability of larvae to find local and remote light sources. Using high speed recording, we reveal the swim motor patterns associated with arearestricted search and roaming, and show that arearestricted search is generated by utilizing an efficient helical swim pattern composed of same direction turns. Finally, we establish that initiating area-restricted search requires retinal input while the deep brain photoreceptor opn $4 a$ and otpa-specified somatostatin releasing neurons control the transition from intensive local search to a roaming state.

\section{Results}

\section{Helical swimming follows loss of illumination}

The visual motor response is a 5- to 10-minute period of hyperactivity that occurs after loss of illumination [24]. In previous studies, the visual motor response has usually been elicited in larvae confined in multi-well plates, precluding the observation of a possible spatial structure in the response. Therefore, to determine whether movement shows a specific spatial pattern or is undirected, we tracked the three-dimensional swim paths of larvae in a large volume chamber $(85 \times 85 \times 75 \mathrm{~mm}$ in length, width, and height, respectively) (Fig. 1a). During full-field illumination, locomotor trajectories were primarily in the $\mathrm{X}-\mathrm{Y}$ plane with relatively little vertical displacement (Fig. 1b, c, baseline). Conversely, during the first minute after loss of illumination, larvae increased downward movement and reduced net displacement in the $\mathrm{X}-\mathrm{Y}$ plane (Fig. 1b, c, local). These changes reduce net threedimensional displacement compared to baseline (baseline, $45.5 \pm 7.58 \mathrm{~mm}$ per 2-minute time interval; $\mathrm{T}_{0}, 30.9 \pm$ $2.73 \mathrm{~mm}$; t-test $\mathrm{t}_{33}=2.28, P=0.03$ ). Intriguingly, we noticed that descent trajectories had a helical shape, which was especially apparent when the swim path was projected onto the $\mathrm{X}-\mathrm{Y}$ plane (Fig. 1b, Dark $\mathrm{T}_{0}$ ). After $3 \mathrm{~min}$ in sustained darkness, we observed that movements were similar to baseline, again primarily directed in the horizontal plane (Fig. 1c, Dark $\mathrm{T}_{4}$ ) restoring net three-dimensional displacement $\left(\mathrm{T}_{4}, 49.0 \pm 5.61 \mathrm{~mm}\right.$; t-test vs. baseline, $\left.\mathrm{t}_{21}=0.377, P=0.71\right)$. Thus, initially after loss of illumination, larvae do not move randomly, but show a strong tendency to slowly swim downwards in the water column, within a spatially restricted region in the horizontal plane.

We next separately analyzed the $\mathrm{X}-\mathrm{Y}$ planar and vertical components of the response. Taking the vertical component first, we confirmed a previous observation that larvae tend to swim near the top of the water column, and after loss of illumination, swim downwards [26]. However when tested during prolonged loss of illumination, we noted that the 'diving response' was transient, lasting for around 1 minute before larvae slowly returned to their baseline position in the water column (time to half recovery $=210 \mathrm{~s}$ after light extinction; Fig. 1d). The mean rate of descent following the loss of illumination was $0.75 \pm 0.08 \mathrm{~mm} / \mathrm{s} \quad(\mathrm{n}=54)$, two orders of magnitude slower than the velocity of burst swims $(100 \mathrm{~mm} / \mathrm{s})$ [29], which are a characteristic feature of escape movements in larvae. Downward swimming is therefore unlikely to be part of a defensive response. Thus, in the vertical dimension, the response to loss of illumination has two phases: a slow downwards swim, followed by a gradual return to the water surface.

\section{Local movement shows characteristic features of area-restricted search behavior}

To study responses in the horizontal plane, we used a large shallow arena $(200 \times 200 \times 5 \mathrm{~mm})$ and tracked individual larvae continuously (10 min baseline, $10 \mathrm{~min}$ dark). During baseline conditions, larvae tended to move in long arching paths with relatively low amplitude trajectory changes (Fig. 2a left panel; Additional file 1: Movie 1a). In contrast, after light extinction, larvae initially remained in a restricted area and then gradually transitioned to an outward pattern of swimming similar to baseline (Fig. 2a right panel; Additional file 1: Movie 

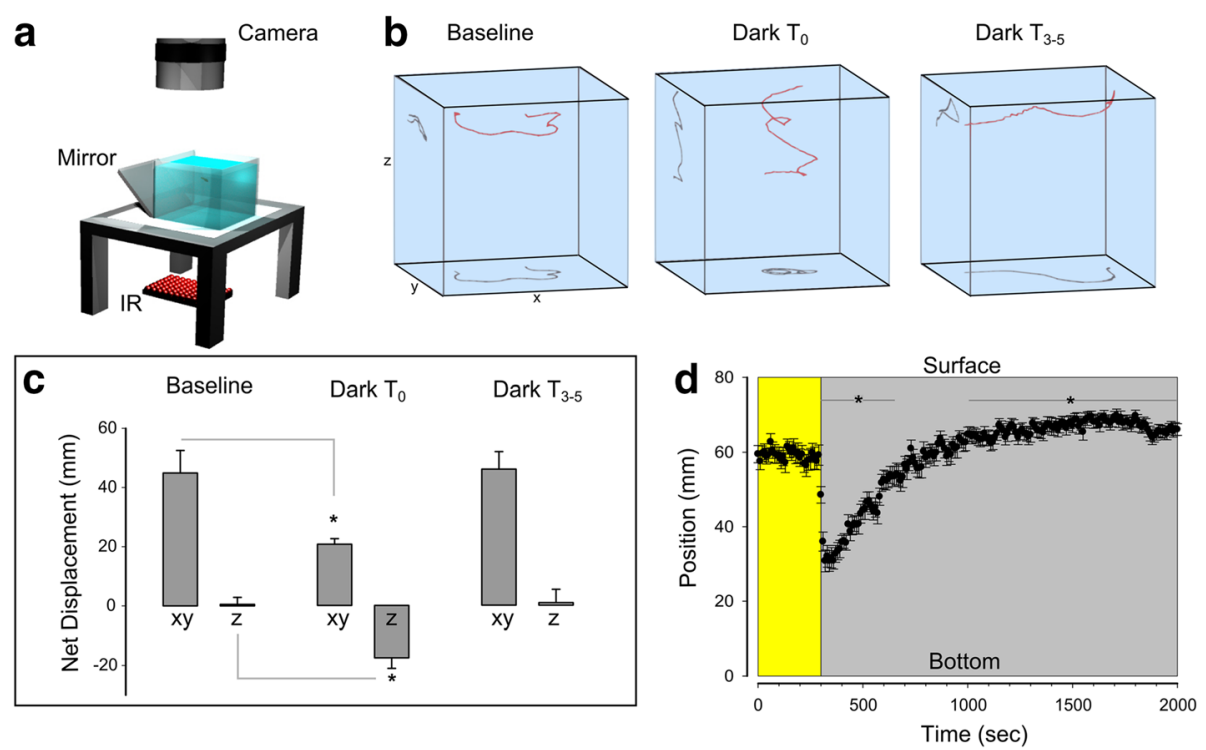

Fig. 1 Changes in 3D swim trajectories after loss of illumination. a Diagram of 3D recording set up. A mirror was positioned adjacent to arena in order to simultaneously view XY and Z planes. b Representative 30-s path trajectories of larvae (red traces) during illuminated baseline conditions (left panel), immediately (middle panel) or 3-5 min after loss of light (right panel). Black traces show 2D projections in YZ and XY planes. Chamber size: $85 \times$ $85 \times 75 \mathrm{~mm}$. c Mean displacement per 30-s recording period in $X Y$ and $Z$ axes during baseline swimming $(N=10)$, during the first minute $\left(T_{0} ; N=25\right)$ and 3-5 min after loss of illumination $\left(T_{3-5} ; N=13\right)$. ${ }^{*} P<0.05$ versus baseline. $\mathbf{d}$ Mean position of larvae in 80-mm deep chamber during baseline illumination (yellow background) and sustained darkness (grey background). $N=30$ groups of five larvae. ${ }^{*} P<0.05$ versus baseline mean

1b). Accordingly, for the first 3 min after the onset of darkness, travel distance was strongly reduced compared to baseline, confirming that movement was spatially restricted (repeated ANOVA $F_{1,172}=2784.7, P<0.001$; Fig. 2b) consistent with reduced displacement in the $\mathrm{X}-$ $\mathrm{Y}$ plane seen in the three-dimensional environment.

Spatially restricted movement forms part of a behavioral strategy that is used by many animals, known as area-restricted search. Area-restricted search occurs during foraging and mate-search behavior in insects, birds and mammals $[2,8,28]$, and during navigation to a shelter or nesting site [30-32]. Characteristic features of area-restricted search are (1) a reduction in travel distance, (2) an increase in movements that cause a change in orientation, (3) an increase in movement path complexity, and (4) a directional bias to movement trajectories $[1,4,33]$. Collectively, these changes allow efficient sampling of the local environment [1]. Similarly, for the first 2-3 min after loss of illumination, in addition to reduced displacement, we found an elevated rate of reorientation (Meander, Fig. 2c) and greater path complexity (Fractal Dimension, Fig. 2d; see Additional file 2: Figure S1 for illustrative examples).

We used two methods to test for directional bias in movement trajectories. First, we noted that, during the initial movement phase, individual trajectories consistently looped either leftward or rightward resulting in a high mean trajectory direction bias (Fig. 2e). Second, we directly measured the frequency of sequential samedirection turning movements. Locomotion in zebrafish larvae consists of discrete maneuvers that are separated by periods of immobility. Under baseline conditions, larvae primarily generate slow swim (scoot) and routine turn (R-turn) maneuver types (Fig. 3a, Baseline), which generate forward propulsion and a change in orientation respectively [29, 34]. Directional bias has been measured using the Lock index (LI), which represents the normalized frequency of sequential maneuvers performed in the same direction [35] (Additional file 3: Figure S2). Consistent with a recent report, we found that under baseline constant illumination, larvae tended to perform sequential swim maneuvers in the same direction, a weak but statistically significant effect [11] (Fig. 3c). For example, during baseline swimming, larvae predominantly executed scoot/scoot maneuver pairs, for which the LI was small, but non-random $(\mathrm{LI}=34$, one-sample t-test vs. $0, \mathrm{t}_{39}=8.36, P<0.001$; Fig. $3 \mathrm{~b}, \mathrm{c}$, baseline). However, during the local movement phase after loss of illumination, there was a 2.4-fold increase in the frequency of R-turn/R-turn maneuver pairs and a two-fold increase in LI, confirming a strong bias in movement direction (Mann-Whitney $U=11.5, \quad P=0.01$; Fig. 3a Dark, d, e). In contrast, during outward swimming, although larvae maintained an elevated rate of R-turn initiation (Fig. 3f), the LI for R-turns declined to baseline levels (Fig. 3g), consistent with the sustained high 


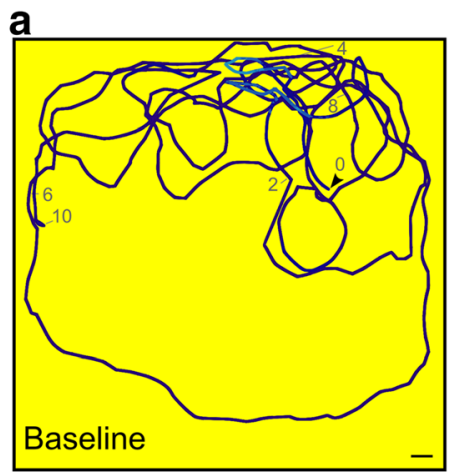

b
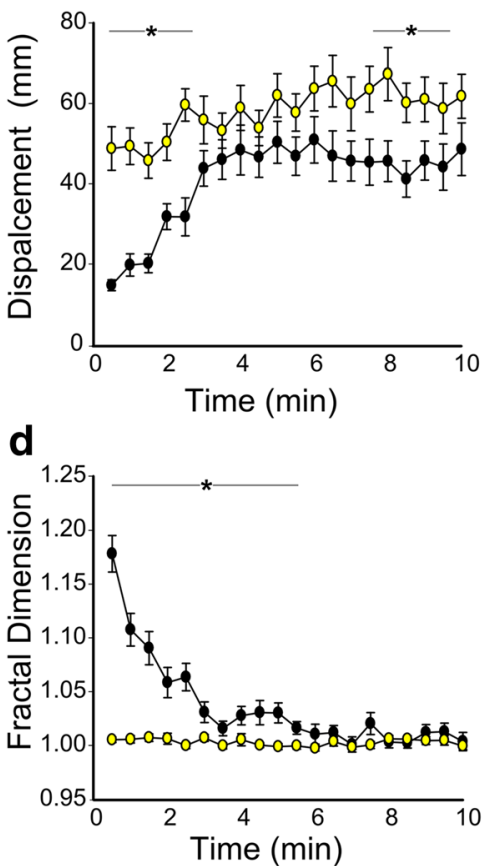

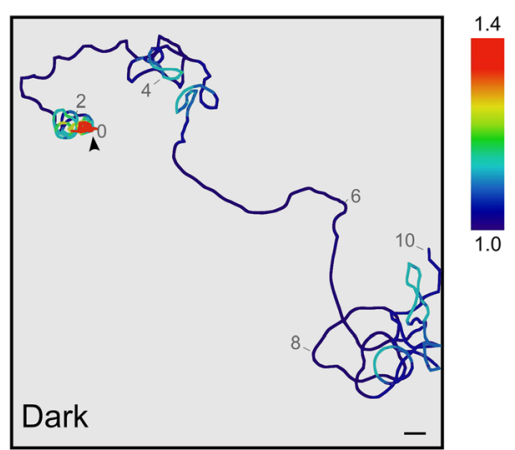

C
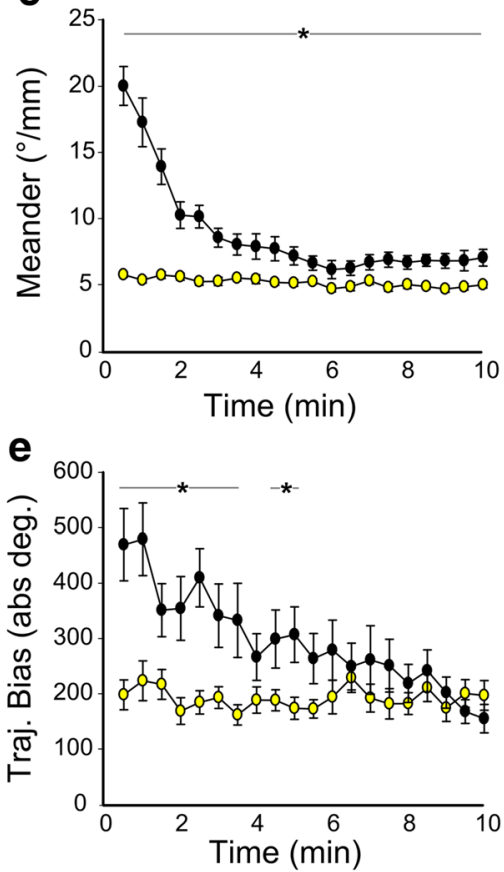

Fig. 2 Locally restricted swimming after loss of illumination. a Representative path trajectories for a larva during full field illumination (left panel) and during the first $10 \mathrm{~min}$ after loss of illumination (right panel). Arrowheads denote starting position and numbers indicate time (min). Color scale indicates fractal dimension in $30 \mathrm{~s}$ windows. Main scale bar $10 \mathrm{~mm}$, chamber is $200 \times 200 \mathrm{~mm}$. Arrowhead length equivalent to larva length. $\mathbf{b}-\mathbf{e}$ Displacement, meander, fractal dimension, and trajectory bias for larvae measured during 10 min of full field illumination (yellow), followed by 10 min darkness (black). $N=32 .{ }^{*} P<0.05$, paired t-test compared to corresponding baseline time-point. See Methods for description of measurements

frequency of re-orienting and loss of directional bias measured using trajectory analysis (Fig. 2c, e). Thus, for the first few minutes after loss of illumination, swim trajectories show a strong directional bias and thus manifest four salient characteristics of area-restricted search behavior.

As for other short-term internal states, search behaviors are typically time-limited and reversible [10]. If a resource is not located within the local environment, a roaming search strategy is often initiated, which is characterized by an outward movement profile [36, 37]. Similarly, spatially restricted movement by larvae persisted only for about 2 min after loss of illumination, after which movement parameters transitioned toward baseline levels. Intriguingly though, two measures, namely displacement and the rate of reorientation, did not match baseline levels even after $10 \mathrm{~min}$ (Fig. 2b, c). Principal component analysis confirmed that trajectories in the first minute after loss of illumination were distinct from baseline movements (Additional file 4: Figure S3a), whereas trajectories after $10 \mathrm{~min}$ sustained dark did not form a separate group but distributed with either the baseline or first-minute dark response trajectories. Thus, the apparent gradual change in mean trajectory parameters after loss of illumination may in fact be due to changes in the frequency with which larvae switch between discrete episodes of spatially restricted movement, and a movement profile similar to baseline (Additional 
a

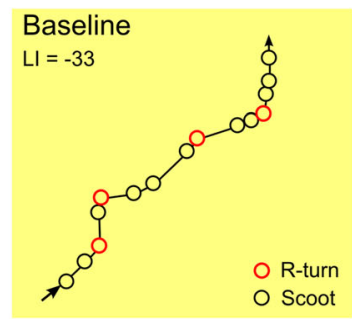

b

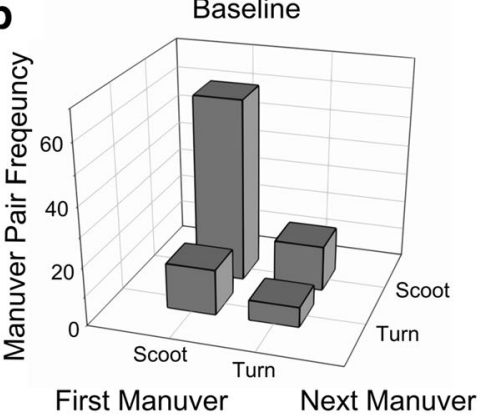

d

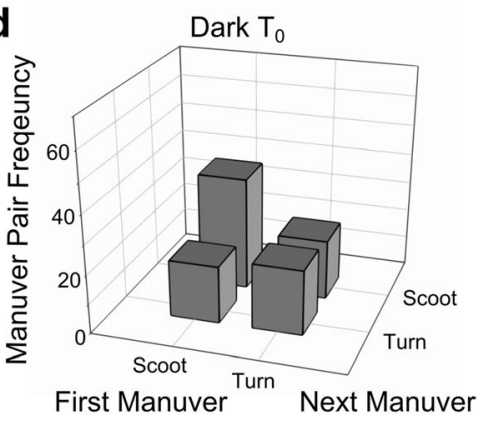

f

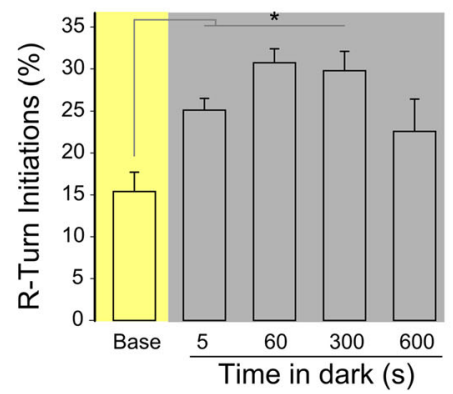

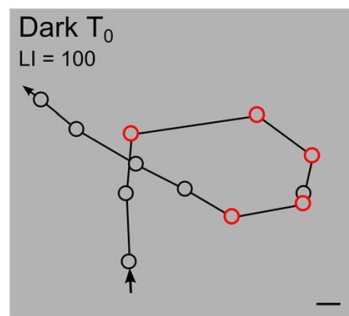

C Baseline

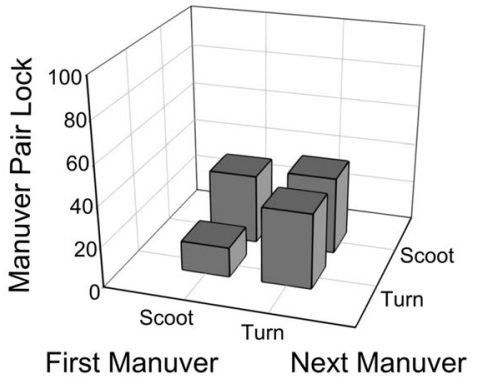

e

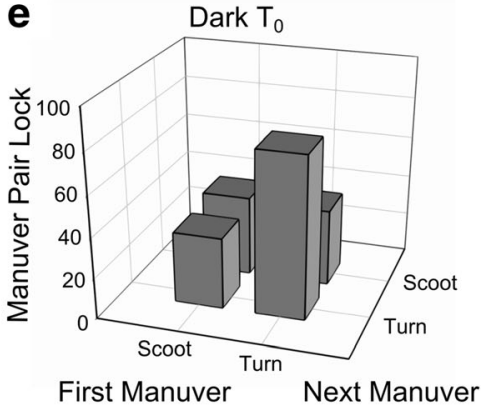

g

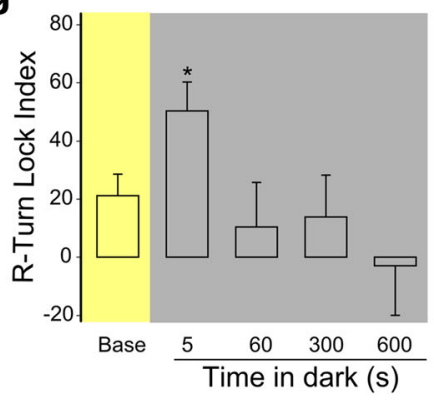

Fig. 3 Local search is generated by increased utilization of same direction R-turn maneuvers. a Representative 10-s path trajectories of individual larva during baseline (left, yellow) and dark (right, grey) conditions. Arrows indicate path direction of the larva. Scoot (black circle) and R-turn (red circle) maneuvers are indicated along each path. Lock index (LI) for each recording noted at bottom. Scale bar 2 mm. Frequency (b, d) and LI (c, e) of maneuver pairs during full-field illumination ( $\mathbf{b}, \mathbf{c}$; baseline) and during the first $10 \mathrm{~s}$ after loss of illumination $\left(\mathbf{c}, \mathbf{d}_{;} ; T_{0}\right)$. Baseline $N=39, T_{0}$ $N=55$. Maneuver frequency analysis excludes O-bends triggered by the sudden reduction in illumination and infrequent fast burst swims. $\mathbf{c}$ Baseline maneuver pair LIs are significantly increased over 0 for scoot-scoot (one-sample t-test vs. $0, \mathrm{t}_{39}=8.36, P<0.001$ ), turn-turn $\left(\mathrm{t}_{24}=2.44, P=0.022\right)$, and turn-scoot pairs ( $\left.t_{39}=4.51, P<0.001\right)$. Scoot-turn maneuver pair $\mathrm{LI}$ was not significantly different from $0\left(\mathrm{t}_{38}=1.01, P=0.32\right)$. $\mathbf{f}$ Frequency of R-turn initiations (percentage of larvae that execute an R-turn per $400 \mathrm{~ms}$ analysis window). $N=17$ groups of 10 larvae each. ${ }^{*} P<0.001 . \mathbf{g} \mathrm{LI}$ for sequential Rturns during sustained loss of illumination. $N=51$ larvae (baseline), 24 (5 s), 22 (60 s), 15 (300 s), 10 (600 s). * $P<0.05$ compared to baseline time-point

file 4: Figure S3b-e). Nevertheless, episodes of spatially restricted behavior declined in frequency after loss of illumination, confirming that this response is timelimited (Additional file 4: Figure S3f).
Behavioral search states are also terminated if a resource is located. To mimic the effect of a successful search, we restored illumination. All locomotor parameters immediately returned to baseline levels when light 
was resumed either during the local movement phase (two-way ANOVA no main effect of prior dark exposure $\mathrm{F}_{1,135}=2.8, P=0.096$; Fig. 4a, Additional file 5: Figure S4) or during the outward movement phase (Fig. 4b; ttest, no difference between baseline and light-restored rate of reorientation $t_{44}=1.73, P=0.09$ ). Thus, after loss of illumination, larvae manifest a time-limited and reversible behavior in which they initially swim downwards, while repeatedly executing R-turn maneuvers in a spatially restricted region in the horizontal plane, establishing a helical trajectory. This movement profile is similar to area-restricted search, enabling larvae to intensively survey the three-dimensional environment. If larvae do not locate light, then a new movement profile gradually emerges in which R-turn maneuvers remain elevated but are no longer executed continuously in a single direction, thereby generating outward trajectories. These observations suggest that local movement and outward swim trajectories represent area-restricted and remote light-search behaviors, respectively.

\section{Local and outward movement patterns differentially facilitate light-search behavior}

Motivated behaviors such as search-states are goal directed, in that they promote specific objectives [10, 38, 39]. Therefore, to rigorously test whether local and outward movement patterns facilitate light-search behavior, we developed two novel phototaxis assays. First, we established a covert phototaxis assay to test whether spatially restricted movement promotes the discovery of light in the immediate environment. Using real-time tracking we positioned a small ( $7 \mathrm{~mm}$ radius) light spot two body lengths directly behind the larva (Fig. 5a). Because the eyes are positioned laterally and angled slightly forward, the spot was not visible to the larva [40]. We then measured the time taken by larvae to swim into the spot. Larvae reached the spot $34 \%$ more quickly when tested during the local movement phase ( $5 \mathrm{~s}$ after loss of full-field illumination), than when tested during the outward movement phase (3-5 min after loss of illumination; $\mathrm{U}=238.5, P=0.011$; Fig. $5 \mathrm{~b}$, Additional file 6: Movies 2a, b). Accelerated phototaxis during the local movement phase could reflect either increased light sensitivity, or earlier light spot detection due to the high rate of re-orientation. To test visual sensitivity, we measured how accurately larvae turned toward a target light within their visual range [15]. Turn movements were strongly biased toward the light during the local movement phase, but not during the outward movement phase (two-way ANOVA, interaction between movement phase and turn bias, $F_{2,162}=11.5, P=0.001$; Fig. $5 c$, d). Thus, during the local movement phase, larvae not only change orientation more frequently than during the outward movement phase, but also show increased responsiveness to a source of illumination.

We next employed a concealed target assay to determine whether outward movement promotes discovery of light that is not in the immediate environment. Here, we used real-time tracking of larval position to project a light spot on the opposite side of a central barrier. The spot was illuminated either $1 \mathrm{~s}$ or 3-5 min after loss of full-field illumination to test larvae in the local and outward movement phases, respectively. In trials without a central barrier, larvae rapidly navigated to the light spot regardless of the duration of time spent in the dark (Fig. 5e). Conversely, in the presence of the barrier, larvae only initiated phototaxis if they first swam to the edge of the barrier (Fig. 5f). During control trials (no light spot illuminated) larvae infrequently circumnavigated the barrier (Fig 5g, No spot). Similarly, when the
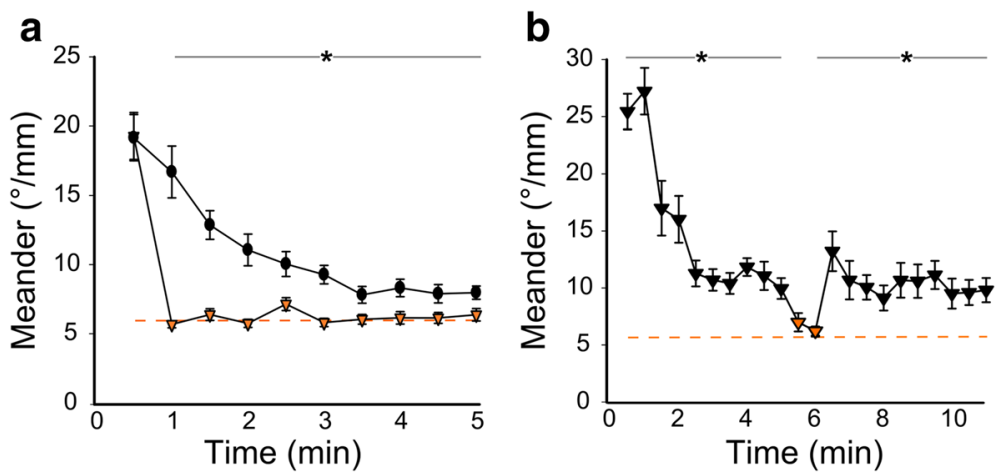

Fig. 4 Light reverses changes in locomotor profiles during local and outward swimming. a Rate of re-orienting (meander) for larvae measured for 5 min after loss of illumination, either in constant darkness (black circles, $N=25$ ), or when illumination was restored after 30 s darkness (orange triangles, $N=22)$. ${ }^{*} P<0.05$ for corresponding time-points in sustained dark and after re-activation of the light. $\mathbf{b}$ Meander for larvae measured for 10 min after loss of illumination, with darkness maintained (black triangles) except during $1 \mathrm{~min}$, when the light was re-activated (orange triangles). $N=19$. ${ }^{*} P<0.05$ compared to constant illumination. Dashed lines in $(\mathbf{a}, \mathbf{b})$ indicate mean meander for larvae measured during constant illumination 


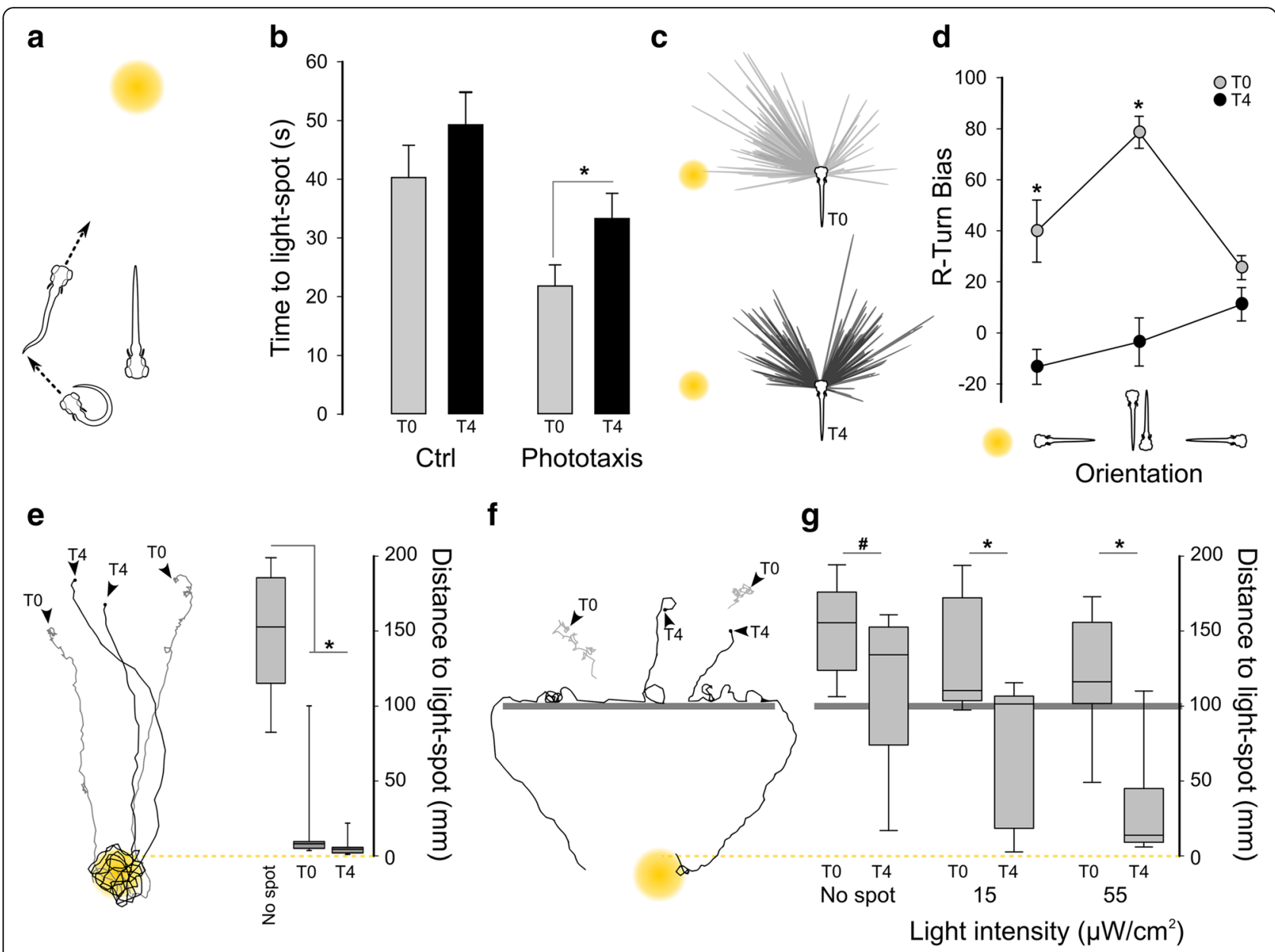

Fig. 5 Local and outward movement strategies differentially facilitate locating local and remote light. a Schematic of covert phototaxis assay. The light spot (14 mm diameter) was projected $7 \mathrm{~mm}$ directly behind a freely swimming larva. We then measured the time for the larva to enter the light spot. b Time to light spot perimeter when activated either $2 \mathrm{~s}\left(T_{0}\right)$ or 3-5 min $\left(T_{4}\right)$ (mean $248 \pm 19.6 \mathrm{~s}$, depending on when larvae entered the motion trigger region of interest (ROI)) after loss of full field illumination. Controls: no target light. $N=33$ ( $T_{0}$ control), 40 ( $T_{0}$ with spot), 18 ( $T_{4}$ control), 20 ( $T_{4}$ with spot). c Turn maneuver trajectories for larvae oriented with the light spot to their right, when tested $2 \mathrm{~s}\left(T_{0}\right)$ or $3-5$ min $\left(T_{4}\right)$ after loss of full-field illumination. $\mathbf{d}$ R-turn direction bias during phototaxis relative to a static light spot. Light spot ( 9 mm diameter, intensity of $20 \mu \mathrm{W} /$ $\mathrm{cm}^{2}$ ) was illuminated either $2 \mathrm{~s}$ (grey; $N=22$ groups of 15 larvae) or $3 \mathrm{~min}$ (black; $N=20$ groups) after loss of full field illumination. Orientation of larvae relative to the target spot is indicated. ${ }^{*} P<0.05$ between $T_{0}$ and $T_{4}$ orientation-matched groups. Bias is the proportion of R-turns directed toward the target spot, normalized between -100 (consistently away from target) to +100 (always toward target). e Phototaxis in a large area (200×200 mm) using a 55- $\mu \mathrm{W}$ light spot. Representative swim trajectories for larvae tested when the light spot was activated $1 \mathrm{~s}\left(T_{0}\right.$, grey traces) or 3-5 min $\left(T_{4}\right.$, black traces) after loss of illumination. Arrowheads indicate start positions. Box plot shows closest approach to the light spot for larvae tested $1 \mathrm{~s}$ ( $T_{0,}$ grey) or 3-5 min (mean $247.7 \pm 21 \mathrm{~s}_{;} \mathrm{T}_{4}$, black) after loss of illumination, and for trials where the light spot was not activated (No spot). $N=11$ larvae (dark, $\mathrm{T}_{0}$ ), 13 (dark, $\left.T_{4}\right)$. f Representative swim trajectories for larvae tested when the light spot was activated $1 \mathrm{~s}\left(T_{0}\right.$, grey traces) or 3-5 minute $\left(T_{4}\right.$, black traces) after loss of illumination. Arrowheads indicate start positions. $\mathbf{g}$ Quantification of concealed target test. Closest approach to the light spot for larvae tested $1 \mathrm{~s}\left(\mathrm{~T}_{0}\right.$, grey) or 3-5 min (mean $236 \pm 13 \mathrm{~s} ; \mathrm{T}_{4}$, black) after loss of illumination with either no target light (No spot), or targets of the indicated intensities. Horizontal black line represents position of barrier. $N=12$ larvae (no spot, $\left.\mathrm{T}_{0}\right), 12$ (no spot, $\left.\mathrm{T}_{4}\right), 10\left(15 \mu \mathrm{W}, \mathrm{T}_{0}\right), 11\left(15 \mu \mathrm{W}, \mathrm{T}_{4}\right), 12\left(55 \mu \mathrm{W}, \mathrm{T}_{0}\right.$ ), and $9\left(55 \mu \mathrm{W}, \mathrm{T}_{4}\right) . \# P<0.05, * P<0.001$ between $\mathrm{T}_{0}$ and $\mathrm{T}_{4}$ groups. Experiment was performed as for $(\mathbf{e})$ except with an interior barrier

spot was illuminated $1 \mathrm{~s}$ after loss of full-field illumination, larvae did not reach the edge of the barrier and therefore did not initiate phototaxis (Fig. $5 \mathrm{~g} ; \mathrm{T}_{0}$ ). In contrast, most larvae tested 3-5 min after loss of full-field illumination swam to the edge of the barrier, then quickly navigated to the light spot (Fig. $5 \mathrm{~g} ; \mathrm{T}_{4}$ ). The difference in final proximity to the light spot was significant (ANOVA, main effect of time in dark $F_{1,38}$
$=36.3, P<0.001)$, demonstrating that larvae locate remote light sources more rapidly during the outward movement phase.

During the concealed target assay, larvae rarely swam continuously beside the barrier, but often appeared to contact it then move away. This was surprising because several studies have argued that zebrafish larvae perform thigmotaxis (touch-seeking) behavior [34, 41]. However, 
supporting experiments used an arena with square or concave walls where it is difficult to distinguish true thigmotaxis from mere wall-following due to outward swim trajectories. In fact, under baseline conditions, larvae tended to swim in close contact with the walls of a concave arena but did not show a significant preference to swim in contact with a convex barrier (Additional file 7: Figure S5a-c), indicating that continuous contact with the perimeter of a chamber is a trivial consequence of outward movement trajectories. In contrast, after loss of illumination, larvae tended to avoid the edges of the concave arena (repeated measures ANOVA main effect of illumination on wall proximity $F_{1,14}=49.4$, $P<0.001$; Additional file 7: Figure S5d, e). Local and outward movement phases are thus associated with wallavoidance behavior.

\section{Retinal signaling is required to initiate the local movement response}

Larval zebrafish detect and respond to changes in illumination using retinal signaling and intrinsically light sensitive central neurons [25, 26, 42-45]. In particular, both retinal and non-visual photoreceptors drive increased activity after loss of illumination [26]. To assess retinal signaling, we enucleated larvae, then performed kinematic and trajectory analysis. After loss of illumination, the R-turn LI for enucleated larvae increased, confirming a role for non-retinal photoreceptors (Fig. 6a, Enuc). However, the LI was $48 \%$ reduced compared to intact controls, and trajectory analysis indicated that the increase in locked R-turns was insufficient to drive locally restricted movement (repeated measure ANOVA, no main effect of enucleation on fractal dimension baseline vs. dark, $\mathrm{F}_{1,66}=1.06, P=0.31$; Fig. $6 \mathrm{~b}$, Additional file 8:
Figure S6). In contrast, after loss of illumination, the rate of re-orientation for enucleated larvae immediately increased to the level produced by controls during the outward movement phase (Fig. 6c, d). Ablating the pineal complex had no effect on local or outward movement parameters (Additional file 9: Figure S7). Therefore, retinal signaling is required to initiate the local movement phase but extra-retinal signaling mechanisms outside of the pineal are sufficient to drive outward swimming behavior.

\section{Orthopedia-specified somatostatin-releasing neurons and melanopsin are necessary for search strategy transition} Hyperactivity after loss of illumination is impaired in the otpa mutant, a null for the orthopediaA homeobox transcription factor [26]. After loss of illumination, the Rturn LI in otpa mutants was significantly greater than for controls (Fig. 7a) and, accordingly, locally restricted movement was strongly potentiated and persisted for significantly longer, with the movement path complexity remaining elevated throughout the 10-min recording period (two way repeated ANOVA main effect of genotype on fractal dimension $\mathrm{F}_{1,58}=17.65, P<0.001$; Fig. $7 \mathrm{~b}$, c). Thus, in the absence of otpa the local movement phase is potentiated and the transition to outward swim trajectories is impaired.

Enucleated otpa mutants did not react to the loss of illumination, confirming that retina- and otpa-specified neurons account for all pathways required to initiate local and outward movement profiles (Additional file 10: Figure S8, see legend for statistics). We therefore asked which otpa-specified neurons drive transition to the outward movement phase of the response. Otpa specifies multiple neuronal cell types within the larval zebrafish
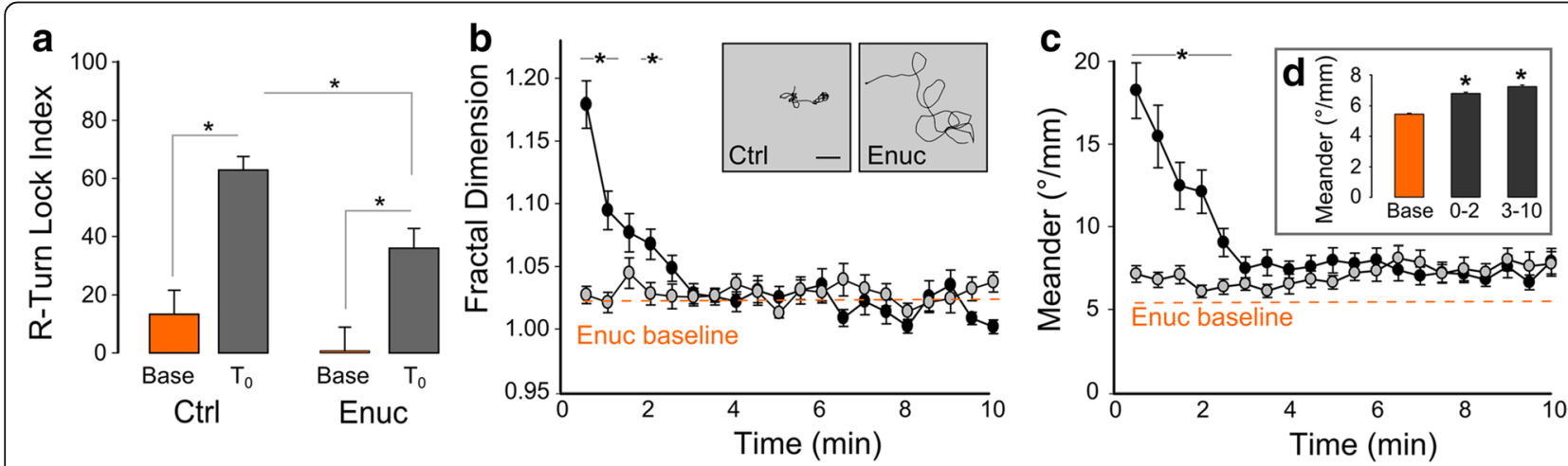

Fig. 6 Retinal input is required to initiate local but not outward movement. a Lock index for R-turns during baseline and immediately following loss of light ( $T_{0}$ ) during $10 \mathrm{~s}$ recordings, for sham operated (ctrl) and enucleated (enuc) larvae. $N=41$ larvae (ctrl, baseline), 53 (ctrl, $T_{0}$ ), 44 (enuc, baseline), 59 (enuc, $\left.T_{0}\right)$. ${ }^{*} P<0.001$. b, c Fractal dimension (b) and meander (c) of path trajectories during dark response for control (black circles, $N=29$ ) and enucleated larvae (grey circles, $N=34$ ). ${ }^{*} P<0.05$ for control compared to enucleated larvae. Dashed lines show mean values for enucleated larvae under full-field illumination. Inset: Representative traces of first 2 min of dark for control (left) and enucleated (right) larvae. Scale bar $20 \mathrm{~mm}$. d Mean meander for enucleated larvae during full-field illumination (base) compared to the first 2 min after loss of illumination (0-2) and from 3-10 min after loss of illumination (3-10). * $P<0.001$, paired t-test compared to baseline 


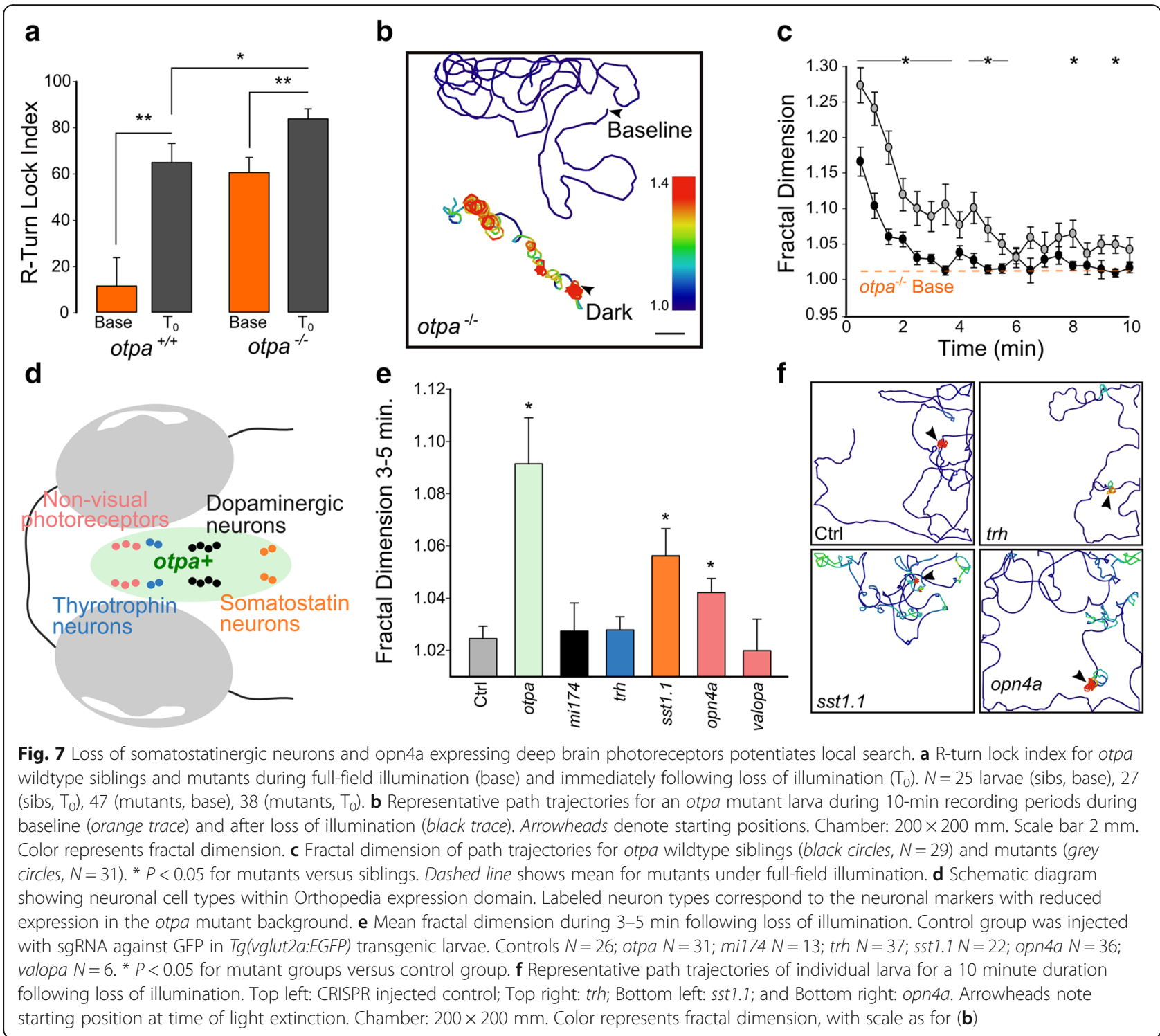

brain, including ventral diencephalon dopaminergic neurons (DA), and thyrotropin- (trh) and somatostatinreleasing neurons (sst1.1) (Fig. 7d) [46]. We tested the contribution of these cell types using mutant lines, and by directly generating homozygous mutant larvae using CRISPR [47]. For CRISPR mutants, we controlled for off-target cutting by replicating our results using independent guide RNAs against different loci within the target genes. Loss of sst1.1 recapitulated the delay in swim trajectory transition observed in otpa, whereas no significant changes in locomotor output were observed after loss of DA neurons or trh neuropeptide (Fig. 7e, f, Additional file 11: Figure S9). Otpa mutants also lose light-sensitive melanopsin (opn4a)-expressing neurons in the anterior preoptic region [26]. Accordingly, opn4a mutants exhibited a delayed transition to outward swim trajectories, whereas transition was normal after loss of the non-visual opsin valopa, which is not specified by otpa (Fig. 7e, f, Additional file 11: Figure S9). Thus, among otpa-specified neurons, somatostatinergic neurons, and non-visual opn $4 a$-expressing photoreceptors drive the transition from local to outward swimming movements (Fig. 8).

\section{Discussion}

We previously proposed that the hyperactivity manifest by larvae in the minutes after loss of illumination promotes aggregation in illuminated regions via photokinesis. Photokinesis is a simple behavioral strategy for navigation that uses rapid non-directional movement in dark areas and slower movement in illuminated regions. However, we now demonstrate that the locomotor 


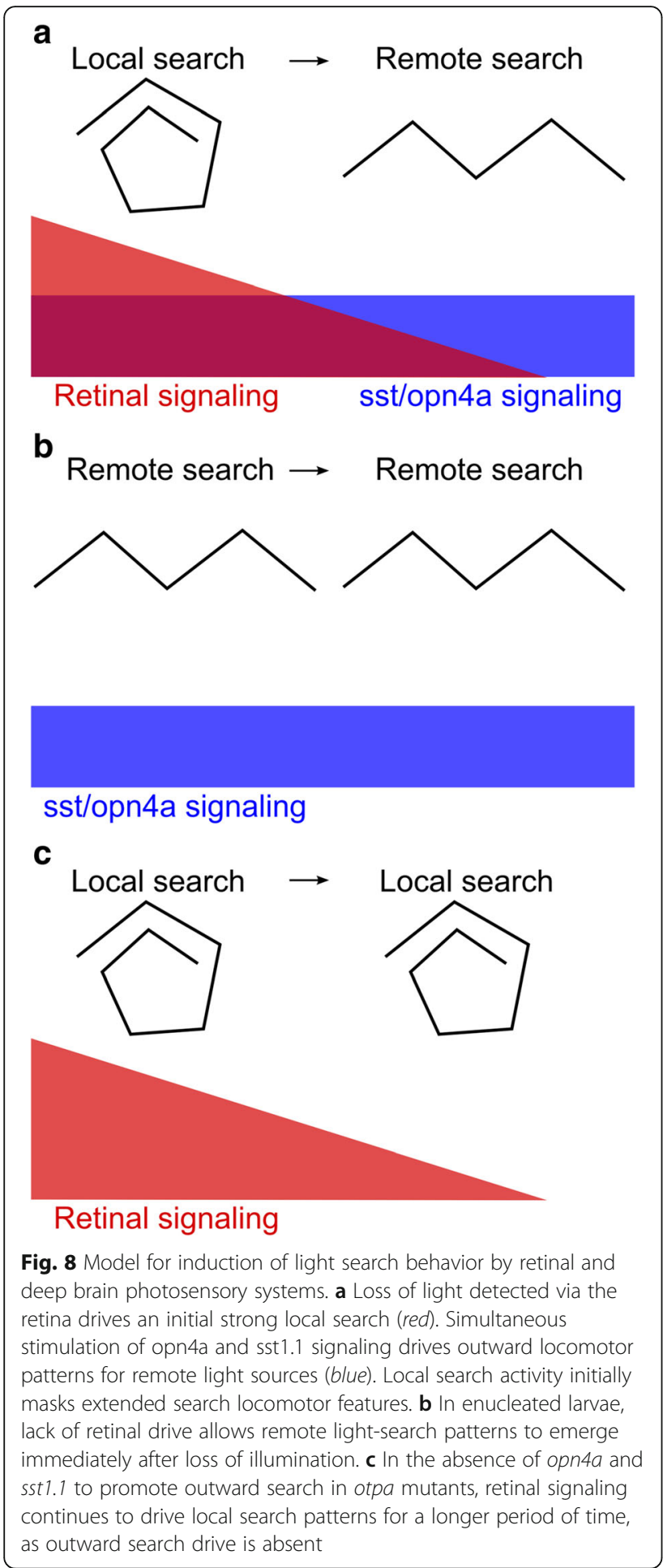

activity after loss of illumination is not undirected. Rather, movement trajectories are highly spatially structured and change during the first few minutes in darkness. For the first 1-2 min after loss of illumination, larvae turn intensively to the same side in the horizontal plane, while gradually swimming downwards, resulting in a helical trajectory. After $3 \mathrm{~min}$, larvae continue to execute re-orienting movements at high frequency, but no longer continuously in the same direction; swim trajectories thus propel larvae out of the local environment.

We considered various interpretations of these movement profiles. For instance, light extinction may trigger an aversive or flight response. Indeed, larvae perform a vigorous $\mathrm{O}$-bend response in the first second after loss of illumination [25]; however, because swim travel distance thereafter diminishes below baseline levels, local and outward swim profiles are inconsistent with escape behavior. Alternatively, the high fractal dimension and slow descent during the local movement phase might suggest a disorganized or confused behavior, but this is contraindicated by the highly-accurate light-orientation behavior, efficient phototaxis, stable same-side turning and wall-avoidance that also occur during this period. Finally, these movement profiles are inconsistent with behavioral sleep, which includes reduced sensory thresholds and substantial reductions in locomotor activity [23].

Rather, we interpret the local and outward movement phases as sequential light-seeking responses. Initially, larvae execute an area-restricted light-search behavior, which if unsuccessful, is followed by a remote lightsearch behavior. Multiple lines of evidence support this view. First, swim trajectories during the local movement phase (reduced travel distance, increased meander, fractal dimension and directionality) are similar to characteristic features of area-restricted search. Similar three-dimensional helical foraging trajectories have been documented in Drosophila and microzooplankton and proposed to yield an optimal search strategy when searching for sparsely distributed food sources [48, 49]. Second, changes to swim profiles were time-limited and rapidly reversed upon restoring illumination, consistent with other motivated states. Third, as for other shortterm internal states [20,21], larvae manifest changes in responsiveness to sensory cues, namely increased sensitivity to a light-spot during the local movement phase, and greater wall avoidance during both local and outward movement phases. Finally, functional tests of lightseeking behavior support this interpretation - larvae more rapidly reached a nearby patch of light during the local movement phase, and conversely located distant illuminated regions only when tested during the outward movement phase. These changes fulfill operational criteria for defining motivated states [10], in that loss of light produces a change in behavior that (1) is maintained internally, (2) is reversible, and (3) facilitates a specific objective. We therefore propose that the swim profiles seen after loss of illumination constitute internally driven search behaviors that enable larvae to efficiently navigate back into illuminated regions. During 
active phototaxis, movements that re-orient larvae away from a light-spot are rapidly corrected by contraversive turns $[15,35]$. However, in the natural environment, a more extensive area-restricted search may be triggered after larvae swim into a small patch of thick foliage or if light is obscured by falling debris. In contrast, sustained remote search activity may be deployed after water currents sweep larvae into large regions of dense undergrowth.

Locomotor responses to loss of illumination are driven by both retinal signaling and otpa-specified deep brain photoreceptors [26]. Our results now reveal that retinal signaling and deep brain photoreceptors differentially trigger local and roaming search states; retinal signaling is required for local movement, whereas otpa-dependent sst1.1-expressing neurons and opn4a-expressing deepbrain photoreceptors sustain roaming search behavior. However, search strategy transition was less impaired in sst1.1 and opn $4 a$ mutants than in otpa mutants. Possible reasons are the presence of supernumerary oxytocin expressing neurons in otpa mutants [46], and the presence of 32 nonvisual opsins in the zebrafish [50], raising the potential for uncharacterized additional photoreceptive neurons in the otpa expression domain. Nevertheless, our data suggests that after loss of illumination, retinal signaling activates an intense area-restricted search, after which sst1.1- and opn4a-dependent pathways gradually drive the transition to a roaming search state. Indeed, somatostatin signaling regulates other motivated behaviors, tuning response thresholds during reward [51, 52] and defensive [53] behaviors in mammals. In our current model, otpa neurons provide a relatively weak drive, which predominates only when the local search state declines. This idea is supported by the finding that enucleated larvae acutely increase re-orientation rates immediately after the loss of illumination. Somatostatin and opn 4 a may therefore continuously drive the roaming search state in darkness, an impulse which is normally masked by retinal-driven local search. Conversely, in the absence of otpa circuitry, retinal signaling is unimpeded by remote search drive, prolonging the local response.

Light-search behavior is likely impelled by dwindling nutritional resources. By 6 dpf, yolk supplies are almost depleted and larvae actively pursue prey. Predation requires visual input; larvae are almost completely unable to locate food in darkness [54]. Accordingly, larvae are hard-wired to seek and remain in illuminated regions, demonstrating vigorous phototaxis behavior toward restricted areas of light. During phototaxis, sensory information signals through retinal ON and OFF pathways to trigger forward swims and turns, respectively [15]. Our new results reveal that, in the absence of directional light cues, loss of illumination triggers sequential internally driven light-search strategies involving, first, an area-restricted search for light in the immediate vicinity, followed - if unsuccessful - by a roaming search for light in distant regions.

An unresolved question concerns the "baseline" state of larvae. After 1-2 min of local search, larvae continue executing turn movements at a high rate, but no longer strongly biased to one side, thereby causing outward swimming. Although functionally, outward swimming enabled larvae to locate remote light sources more quickly than during the local movement phase, principle component analysis indicated that outward movement parameters clustered either with local search or baseline swim profiles. Thus, the outward swimming phase, while occasionally interrupted by episodes of local search behavior, showed similar trajectory characteristics to baseline activity under full-field illumination. Nevertheless, wall-avoidance and meander measurements revealed differences between baseline and outward swimming, indicating distinct behavioral states. A strong possibility is that "baseline" behavior under full-field illumination is actually a food-search state; unfed $6 \mathrm{dpf}$ larvae, their yolk supplies exhausted, may already be in a roaming search for food, an idea which is consistent with recent work analyzing movement under constant illumination [11] and changes in sensory responsiveness after feeding [20].

Unexpectedly, net locomotor activity decreased after a loss of illumination. Reduced activity contrasts sharply with many studies on the visual motor response, including from our laboratory, reporting that loss of illumination triggers 5-10 min of hyperactivity $[26,55,56]$. We speculate that chamber size accounts for the discrepancy. In most previous work, movement has been constrained using small wells in which larvae constantly encounter the edge of the arena. However, we found that after loss of illumination, larvae avoid barriers, suggesting that in small arenas such as multi-well plates, where the perimeter is frequently encountered, wall avoidance may trigger increased activity. The rationale for wall avoidance after loss of illumination remains unclear, possibly reflecting a strategy to reduce collisions in darkness, or alternatively facilitating exploration by moving larvae away from barriers that prevent them from detecting distant light sources.

\section{Conclusion}

Larval stage zebrafish larvae maintain distinct behavioral states, including arousal and sleep [10, 20-22]. Our new findings reveal that internal drives in larvae can additionally produce sophisticated motivated behaviors such as search activity. It is likely that larvae exhibit additional types of autogenic search behavior provoked by exposure to, or loss of stimuli in other sensory modalities. The 
finding that non-retinal photoreception by melanopsin and somatostatin signaling play a selective role in driving remote light-search is consistent with the idea that nonvisual photoreceptors are intimately linked to motivational state control [57-59], and provides the opportunity to dissect the ancient evolutionary pathways that allow organisms to adapt to short-term challenges and opportunities in the dynamic natural environment.

\section{Methods}

\section{Zebrafish husbandry}

Zebrafish were maintained on a Tubingen long fin strain background. Larvae were raised at $28{ }^{\circ} \mathrm{C}$ in E3 medium, under Light:Dark cycle (14:10 h) and maintained at a maximum density of 30 per $6-\mathrm{cm}$ Petri dish. otpa ${ }^{m 866}$ homozygous mutant and cousin wildtype pairs (for control experiments) were genotyped as previously described [26]. To test the effect of DA neuron loss we used the previously established mi174 mutant, which has a global loss or dysfunction of dopaminergic neurons, including Orthopedia-specified DA neurons [60, 61]. Homozygous mutant mi174 larvae (null for $\operatorname{trpM7)}$ were identified by the hypopigmentation phenotype and normally pigmented siblings from the same clutches were used for controls [62] (kind gift from Jim Hudspeth lab). Enucleations were performed on $4 \mathrm{dpf}$ larvae that were anaesthetized in Evans solution with tricaine. Using a Sylgard-coated dish with a groove to steady the larva, both eyes were removed with sharp forceps. After enucleation, larvae were allowed to recover in fresh Evans solution $(134 \mathrm{mM} \mathrm{NaCl}, 2.9 \mathrm{mM} \mathrm{KCl}$, $2.1 \mathrm{mM} \mathrm{CaCl}_{2}, 1.2 \mathrm{mM} \mathrm{MgCl}, 10 \mathrm{mM}$ glucose and $10 \mathrm{mM}$ Hepes $\mathrm{pH}$ 7.8). After approximately $24 \mathrm{~h}$ larvae were transferred to E3 media. Control larvae were treated with tricaine and incubated in Evans solution in parallel with enucleated larvae. Enucleation produced characteristic melanophore expansion and a complete loss of high angular O-bend responses in response to a dark flash [63], confirming that larvae were functionally blind (not shown). All in vivo experimental protocols were approved by the NICHD animal care and use committee.

\section{Behavioral testing}

Tests were performed on larvae at 6 or $7 \mathrm{dpf}$. Behavioral assays were monitored using infrared illumination (CMVision Supplies, $850 \mathrm{~nm}$ ) positioned below the chamber, with IR-longpass filters on the camera lens to exclude visible light. For white-light illumination we used LEDs positioned above the chamber unless otherwise specified. Prior to behavioral recording larvae were adapted for a minimum of 30 min to the same light intensity as in the testing arena. Light intensities were measured using a radiometer (International Light Technologies).
Testing arenas were maintained at $28{ }^{\circ} \mathrm{C}$. Image acquisition and illumination were controlled using DAQtimer event control software [63]. We added new motion trigger code to DAQtimer to detect when a larva entered a specified ROI. For kinematic analysis, we recorded video at 1000 frames per s (fps) using a Fastcam 1024 (Tech Imaging). Tracking was performed offline using Flote tracking software, which also measured swim kinematics and classified locomotor maneuvers [63]. For trajectory analysis (all recordings at less than $1000 \mathrm{fps}$ ) images were captured with a $\mu$ Eye IDS-1545LE-M CMOS camera $\left(1^{\text {st }}\right.$ Vision, Andover, MA), tracked in real-time using DAQtimer [21], and analyzed offline using custom scripts written in IDL (Exelis, Boulder, CO).

\section{D trajectory analysis}

Single larvae were placed in an $85 \times 85 \times 85 \mathrm{~mm}$ clear acrylic chamber filled to a depth of $75 \mathrm{~mm}$, illuminated at $50 \mu \mathrm{W} / \mathrm{cm}^{2}$. Images were captured at $20 \mathrm{~Hz}$ with the recording window adjusted to simultaneously record the $\mathrm{X}-\mathrm{Y}$ plane and the vertical $\mathrm{Z}$ position of the larvae using a mirror placed adjacent to the chamber at a $45^{\circ}$ angle. Each larva was adapted to the arena for at least $5 \mathrm{~min}$ and recordings were started when the larva was centrally positioned in the arena. Tracking was performed offline and horizontal and vertical displacement analyzed in 30$\mathrm{s}$ time windows.

\section{Dive analysis}

Groups of five larvae were placed in a $40 \times 45 \times 100 \mathrm{~mm}$ (length $\times$ width $\times$ height) clear acrylic chamber filled to a height of $80 \mathrm{~mm}$, illuminated at $200 \mu \mathrm{W} / \mathrm{cm}^{2}$. Larvae were adapted to the arena for $10 \mathrm{~min}$ before testing. Recordings were captured at $2 \mathrm{~Hz}$ and included $300 \mathrm{~s}$ of full-field illumination (baseline) followed by $1700 \mathrm{~s}$ in darkness. The mean position of the larvae was analyzed in $2.5 \mathrm{~s}$ bins. We measured descent speed using individual larvae in the same container during the period from 1.6 to $6.0 \mathrm{~s}$ after loss of illumination to capture the linear portion of the response (i.e. after initiation of the downward swim but before larvae reached the bottom of the arena).

\section{D trajectory analysis}

Single larvae were placed in a $200 \times 200 \mathrm{~mm}$ chamber ( $5 \mathrm{~mm}$ depth, illumination $50 \mu \mathrm{W} / \mathrm{cm}^{2}$ ). After a $10 \mathrm{~min}$ adaptation period, recordings were initiated once the larva entered a $120 \times 120 \mathrm{~mm}$ motion trigger ROI in the center of the arena, after which the larva was tracked at $10 \mathrm{~Hz}$ under constant illumination for $10 \mathrm{~min}$. The motion trigger ROI was then restored. Once the trigger was again activated, illumination was turned off and the larva tracked for a second 10-min period. For each larva we then used 30-s track segments to measure displacement, 
distance, meander, fractal dimension, and trajectory bias. Displacement: the straight-line distance between the starting and ending point for each $30 \mathrm{~s}$ interval. Distance: total travel distance, calculated as the total of the straight-line distances covered in each of the thirty $1 \mathrm{~s}$ line segments. Meander: a measure of the rate of reorientation [4], calculated using the total of the absolute values of angles formed by all trajectory changes during the $30 \mathrm{~s}$ interval, divided by the distance. Fractal dimension: a measure of path complexity [64] frequently used in studies of search behavior $[28,49,65]$, which we implemented using the box-counting method [66]. In brief, the fractal dimension was estimated as the slope of the line fitting the log ratio of the number of box units that cover part of the trajectory versus box size. Trajectory bias: a measure of the directional (left or right) bias of the trajectory, calculated using the absolute value of the total of the angles formed by all trajectory changes during the 30-s interval. Leftward and rightward trajectory changes produced negative and positive angles, respectively; thus, these changes summed to near zero when larvae swam in predominantly straight lines. As tracking was not reliable when larvae were adjacent to the wall of this arena, we excluded segments that included less than $10 \mathrm{~s}$ of data. Principal component analysis was performed on measures of displacement, meander, fractal dimension, and trajectory bias after standardization to unit variance and mean-subtraction.

\section{Covert phototaxis assay}

To measure how quickly larvae swam to a nearby light source, we used a $58 \times 58 \mathrm{~mm}$ chamber (depth $5 \mathrm{~mm}$ ), illuminated overhead at $50 \mu \mathrm{W} / \mathrm{cm}^{2}$. Single larvae were placed in the arena, adapted for $2 \mathrm{~min}$ and tracked in realtime. After the larva swam into a $20 \times 20 \mathrm{~mm}$ motion trigger ROI in the center of the arena, full-field illumination was extinguished. After either $3 \mathrm{~s}$ or $3 \mathrm{~min}$ in darkness, we established a second motion trigger in the center of the arena. Once this was triggered we used the position and orientation of the larva (from real-time tracking) to project a stationary 14-mm diameter light spot of $23 \mu \mathrm{W} /$ $\mathrm{cm}^{2}$ intensity at a position $7 \mathrm{~mm}$ directly behind the tail of the fish (AAXA P2 Pico Projector). We recorded the response of the larva, and the position of the light-spot using a second camera with an IR-blocking filter at $60 \mathrm{~Hz}$, then measured offline the time for the larva to enter the perimeter of the spot. During "no target" control experiments the light spot was flashed on for $50 \mathrm{~ms}$, then extinguished, so that we could calculate the time taken for the larva to swim into an equivalent target region by chance. Larvae reached the target spot significantly more quickly when it remained illuminated, confirming that efficient phototaxis occurred (ctrl vs. phototaxis: for $\mathrm{T}_{0}, \mathrm{t}_{56.6}=2.8$, $P=0.007$; for $\mathrm{T}_{4}, \mathrm{t}_{36}=2.5, P=0.016$ ).

\section{Concealed target assay}

The efficiency of locating a light source that was not immediately visible was tested using a $200 \times 200 \mathrm{~mm}$ chamber ( $5 \mathrm{~mm}$ depth, illumination $\left.50 \mu \mathrm{W} / \mathrm{cm}^{2}\right)$. Two LEDs were positioned beneath the chamber on opposite sides, each with an iris to produce a $25-\mathrm{mm}$ light spot on a diffuser immediately below the chamber. We first confirmed that larvae performed robust phototaxis to a light spot illuminated on the opposite side of the chamber (Additional file 4: Figure S3). Then, to prevent larvae from directly detecting the position of a light spot on the opposite side, we added a $140-\mathrm{mm}$ opaque barrier into the middle of the arena. After $10 \mathrm{~min}$ adaptation to the arena, we established a rectangular motion trigger ROI in the center of the arena $(20 \mathrm{~mm}$ away from the ends of the barrier, encompassing regions on both sides of the barrier). After the trigger was activated, full-field illumination was turned off. Next, either $1 \mathrm{~s}$ or $3-5 \mathrm{~min}$ after the onset of darkness, a light-spot was activated on the side of the barrier opposite to the position of the larva. The larva was then tracked for a 2-min period during which we measured the closest position of each larva to the center of the light spot.

\section{Turn bias assay}

Groups of 15 larvae were recorded simultaneously in a 90-mm diameter arena (depth $4 \mathrm{~mm}$, illumination $50 \mu \mathrm{W} / \mathrm{cm}^{2}$ ). After $5 \mathrm{~min}$ adaptation, we extinguished full-field illumination. Then, after either $2 \mathrm{~s}$ or $3 \mathrm{~min}$, we induced phototaxis using a $9 \mathrm{~mm}$ diameter $20 \mu \mathrm{W} / \mathrm{cm}^{2}$ light spot illuminated on a diffuser below one side of the arena and recorded responses for $12 \mathrm{~s}$ with a high speed camera. Turn bias was the tendency of R-turn maneuvers to be made toward the light spot as previously described [15]. Briefly, an index of -100 means all turns are directly away from the spot, and +100 means turns are executed toward the spot. For analysis, larvae were binned based on their orientation relative to the spot.

\section{Kinematic analysis}

To determine maneuver pair frequency and LI we used a $40 \times 40 \mathrm{~mm}$ chamber, illuminated at $200 \mu \mathrm{W} / \mathrm{cm}^{2}$. Single larvae were acclimated in the chamber for $2 \mathrm{~min}$ before a $15 \times 15 \mathrm{~mm}$ motion trigger $\mathrm{ROI}$ in the center of the chamber was established. After the trigger was activated, we collected $10 \mathrm{~s}$ of data using a high speed camera, either maintaining illumination (baseline) or immediately after turning the light off. Larvae were tracked using Flote, then custom scripts were used to calculate the frequency and LI for each maneuver pair. To determine the initiation frequency of R-turns under sustained loss of illumination, experimental parameters were as described above with the following changes. Larvae were recorded in groups of five and acclimated for a period of 
30 min after being placed into the recording arena. Initiation frequency is per $400 \mathrm{~ms}$ analysis window. To measure the R-turn LI during sustained darkness, single larva were recorded at the specified intervals over a 10min time-period. The LI is the percentage of sequential maneuvers that are performed in the same direction, normalized between -100 and +100 , such that random turning has a LI of 0 , whereas for constant same-side turning the LI is +100 (as illustrated in Additional file 3: Figure S2). Only trials in which a larva executed at least three R-turns were used when calculating the LI.

\section{Gene mutations}

Cloning free CRISPRs were designed and synthesized using methods as described [47]. sgRNAs were designed using CRISPRscan [67]. CRISPR sgRNA (120 pg), cas9 RNA (300 pg), and fluorescent protein RNA (30 pg) were co-injected into single cell wildtype embryos. Fluorescent protein RNA (either RFP or GFP) was used as a marker for successful injection and used to sort embryos for behavioral testing. Two-dimensional trajectory behavior and analysis, described above, was performed on successfully injected larvae. CRISPR efficacy was determined for every larva tested using CRISPR-STAT fluorescent PCR (Additional file 12: Figure S10) using an ABI 3100 Genetic Analyzer Avant [68]. Additional file 13: Table S1 lists guide RNA sequences and genotyping primers. Custom software was used to measure percent of wildtype gene target degradation for semi-quantitative analysis of knockdown efficiency. Only larvae with biallelic conversion of the wildtype gene products were included for quantification - a maximum of $5 \%$ detectable wildtype PCR product was used as a threshold for inclusion into the data set. For control experiments, we injected a guide RNA targeting EGFP into in-crosses of a transgenic zebrafish line $T g(v g l u t 2 a: E G F P)$ which was derived from $n n s 14 \mathrm{Tg}$ by Cre injection [69]. Injected control larvae were processed as for experimental groups after assessing loss of EGFP PCR product.

\section{Pineal ablation}

Neuronal ablation was performed as previously described [70]. Briefly, $1 \mathrm{dpf}$ larvae from in-crosses of $y 227 \mathrm{Tg}$, a line with pineal-specific expression of nitroreductase-tagged mCherry, were treated with $10 \mathrm{mM}$ metronidazole in E3 media for $48 \mathrm{~h}$ with drug replacement after $24 \mathrm{~h}$. After treatment, larvae were returned to fresh E3 media. Ablation efficacy was assessed by screening for mCherry fluorescence. Controls were wildtype siblings, also treated with $10 \mathrm{mM}$ metronidazole.

\section{Statistical analysis}

Statistical analysis was performed using SPSS (IBM, Armonk, NY), IDL and Gnumeric (Available from: http://www.gnumeric.org/). Graphs show mean and standard error of the mean unless otherwise specified. Normality of data sets was determined using the Shapiro-Wilk test in SPSS. Significance for normally distributed data sets was calculated using the twotailed Student t-test. For data not normally distributed, the Mann-Whitney U test was applied.

\section{Additional files}

Additional file 1: Movie 1a, b. Representative path trajectory of a larva during (a) baseline illumination and (b) after loss of illumination. Movie duration $10 \mathrm{~min}$. (ZIP $2209 \mathrm{~kb}$ )

Additional file 2: Figure S1. Fractal dimension measurement of path complexity. Illustrative examples of fractal dimension values for $30 \mathrm{~s}$ duration movement paths of increasing complexity. Start position of each path is indicated (black circle). (TIF 503 kb)

Additional file 3: Figure S2. Schematic of lock index measure. Left: random turning produces a Lock index of zero. Right: continuous turning in one direction gives a Lock index of 100. (TIF $352 \mathrm{~kb}$ )

Additional file 4: Figure S3. Periods of area-restricted movement reoccur sporadically during prolonged dark. (a) First three principal components of trajectory measures, which together account for $95.5 \%$ of the variance. Small spheres represent values for 1 minute periods taken from baseline (orange), first minute after loss of illumination (grey) and tenth minute after loss of illumination (blue). (b) Fractal dimension for 10 larva during baseline (orange) and paired dark response (grey). (c) Distribution of fractal dimension values for $30 \mathrm{~s}$ windows during baseline (orange) and dark response (grey). $N=32$ larvae, 40 time-points each. A threshold of 1.10 for local-search behavior (arrow) is exceeded by $0.5 \%$ of traces during baseline recordings, and marks an inflection point in the distribution of values during the dark response. (d) Traces from two larvae over a 10-minute recording after loss of illumination. Boxes indicate period where the fractal dimension of the trajectory exceeded 1.10 during the outward swimming phase of the response. Arrowheads indicate starting locations. (e) Fractal dimension from 3 to 10 min after loss of illumination for the larvae indicated in (b). Red dotted line indicates the threshold value for local-search like behavior. (f) Frequency of ARS in $30 \mathrm{~s}$ time bins, as defined by episodes exceeding the fractal dimension threshold of 1.1. Percent shows proportion of larvae $(N=34)$ manifesting ARS behavior per time-point. (TIF $2394 \mathrm{~kb}$ )

Additional file 5: Figure S4. Light restores baseline trajectory profiles. Fractal dimension (a) and trajectory bias (b) after loss of illumination, either with constant dark (black circles, $N=25$ ) or when full-field illumination was restored after $30 \mathrm{~s}$ of dark (orange triangles, $N=22$ ). Dotted line indicates mean for larvae tested under constant illumination $(N=25)$. ${ }^{*} P<0.05$ for corresponding time-points between larvae in constant dark versus larvae with illumination restored. (TIF $716 \mathrm{~kb}$ )

Additional file 6: Movies $\mathbf{2 a}$, b. Representative response of a larva in the local search assay after (a) a 3 s delay following loss of illumination, and (b) after a 3 minute delay following loss of illumination. (ZIP 6033 kb)

Additional file 7: Figure S5. Wall-avoidance behavior after loss of illumination. Illustrative path trajectories for larvae over 10 min, inside a transparent interior barrier (diameter $85 \mathrm{~mm}$ ) within the recording chamber (a) or outside the barrier (b) under baseline conditions. (c) Time spent within concentric rings of equal area $\left(1000 \mathrm{~mm}^{2}\right)$ progressively further from the interior barrier during 10 min recording as in (b). Differences between time spent in the four rings are not significant (repeated measures ANOVA $F_{3,39}=0.19, P=0.90$ ), indicating that larvae do not show preferential swimming in proximity to a convex wall; $N=14$ larvae. (d) Representative 2 min trajectories for larva inside the interior barrier during baseline (orange), or 8 min after loss of illumination (black). (e) Quantification of (d) - proportion of time spent by larvae within $3 \mathrm{~mm}$ of the wall of the chamber during baseline and at the indicated time-points after loss of illumination; $N=15 .{ }^{*} P<0.05$. (TIF $1499 \mathrm{~kb}$ ) 
Additional file 8: Figure S6. Enucleated larva path trajectories. Representative 10 min trajectory for a control $(a, b)$ and an enucleated larva $(c, d)$ during baseline full-field illumination $(a, c)$ and after loss of illumination (b, d). Arrow indicates starting position of larva at the beginning of the recording. Chamber: $200 \times 200 \mathrm{~mm}$. Color scale represents fractal dimension along path trajectory. (e-f) R-turn and slowswim initiations were not significantly different between control and enucleated larvae after loss of illumination, demonstrating that enucleation did not broadly perturb behavior (independent sample t-test: R-turn $\mathrm{t}(121)=1.5, P=0.129 ;$ Scoot $\mathrm{t}(121)=0.139, P=0.889$ ). (TIF $1543 \mathrm{~kb}$ )

Additional file 9: Figure S7. Response of pineal ablated larvae to loss of illumination. (a) Fractal dimension and (b) meander after loss of illumination in pineal ablated y227Tg larvae (grey, $N=31$ ) and metronidazole treated non-transgenic siblings (black, $N=16$ ). Dashed line shows mean for ablated larvae under full-field illumination. (TIF 1041 kb)

Additional file 10: Figure S8. Response of enucleated otpa mutant larvae to loss of illumination. Displacement (a), path complexity (fractal dimension, b), rate of re-orientation (meander, c), and trajectory bias (d) for enucleated otpa homozygous mutant larvae, recorded for 10 min under full-field illumination (yellow) or following loss of illumination (black); $N=22$. Repeated measures ANOVA showed no significant main effect of enucleation on movement parameters (fractal dimension: $F_{1,38}=0.292, P=0.59$; displacement: $F_{1,40}=0.66, P=0.42 ;$ meander: $F_{1,38}=2.28, P=0.14$; traj. bias: $F_{1,38}=0.087$, $P=0.77)$. (TIF $1296 \mathrm{~kb}$ )

Additional file 11: Figure S9. Confirmation of sst1.1 and opn4a mutant phenotypes using an independent guide RNA. Path complexity for sst1.1 and opn4a mutants generated using independent sgRNAs, targeting different genomic sites than for the experiments in Fig. 7. Quantification is as for Fig. 7e. ${ }^{*} P<0.05$ for mutants versus controls. Controls $N=26$; sst1.1 T2 $N=26$; opn $4 a \mathrm{~T} 2 \mathrm{~N}=43$. (TIF $494 \mathrm{~kb}$ )

Additional file 12: Figure S10. Screening mutant phenotypes by CRISPR-mediated biallelic gene mutation. Representative fluorescent PCR results for amplification around the CRISPR target site in the opn4a gene in: (a) uninjected embryo showing wildtype 144 bp product from opn4a primers. (b) Embryo injected with cas9 and sgRNA against opn4a showing a residual $144 \mathrm{bp}$ peak indicating incomplete mutation of opn4a. Larvae with similar genotypes were excluded from analysis. (c) Injected embryo showing complete loss of the wildtype PCR product, indicating successful biallelic mutation. Peak sizes labeled below each trace. (TIF $1371 \mathrm{~kb}$ )

Additional file 13: Table S1. CRISPR design and genotyping. (XLS 19 kb)

\section{Acknowledgments}

We would like to thank Benjamin Feldman (NICHD) for help with design and analysis of CRISPR experiments.

\section{Funding}

This work was supported by the Intramural Research Program of the Eunice Kennedy Shriver National Institute of Child Health and Human Development.

\section{Authors' contributions}

$\mathrm{EH}$ and $\mathrm{HB}$ conceived and designed the project. $\mathrm{EH}, \mathrm{YB}$ and JS planned and executed experiments. HB wrote software. $\mathrm{EH}$ and $\mathrm{HB}$ wrote the manuscript. All authors read and approved the final manuscript.

\section{Availability of data and materials}

The datasets used and/or analyzed during the current study are available from the corresponding author on reasonable request.

\section{Competing interests}

The authors declare that they have no competing interests.

\section{Consent for publication}

Not applicable.

\section{Ethics approval and consent to participate}

All in vivo experimental protocols were approved by the NICHD animal care and use committee.
Received: 13 December 2016 Accepted: 22 December 2016

Published online: 26 January 2017

\section{References}

1. Bell WJ. Searching behaviour: The behavioural ecology of finding resources. London: Chapman and Hall; 1990.

2. Bell WJ. Sources of information controllling motor patterns in arthropod local search orientation. J Insect Physiol. 1985;31(11):837-47.

3. Wehner R, Srinivasan MV. Searching behavior of desert ants, genus cataglyphis (formicidae, hymenoptera). J Comp Physiol. 1981;142:315-38.

4. White J, Tobin TR, Bell WJ. Local search in the housefly musca domestica after feeding on sucrose. J Insect Physiol. 1984;30(6):477-87.

5. Chalasani SH, Chronis N, Tsunozaki M, Gray JM, Ramot D, Goodman MB, et al. Dissecting a circuit for olfactory behaviour in caenorhabditis elegans. Nature. 2007:450(7166):63-70.

6. Hills T, Brockie PJ, Maricq AV. Dopamine and glutamate control area-restricted search behavior in caenorhabditis elegans. J Neurosci. 2004;24(5):1217-25.

7. Bendena WG, Boudreau JR, Papanicolaou T, Maltby M, Tobe SS, Chin-Sang ID. A caenorhabditis elegans allatostatin/galanin-like receptor npr-9 inhibits local search behavior in response to feeding cues. Proc Natl Acad Sci U S A. 2008;105(4):1339-42.

8. Hills TT, Kalff C, Wiener JM. Adaptive levy processes and area-restricted search in human foraging. PLoS One. 2013;8(4):e60488.

9. Ramos-Fernandez G, Mateos JL, Miramontes O, Cocho G, Larralde H, AyalaOrozco B. Levy walk patterns in the foraging movements of spider monkeys (ateles geoffroyi). Behav Ecol Sociobiol. 2004;55:223-30.

10. Horstick EJ, Mueller T, Burgess HA. Motivated state control in larval zebrafish: Behavioral paradigms and anatomical substrates. J Neurogenet. 2016:30(2): 122-32.

11. Dunn TW, Mu Y, Narayan S, Randlett O, Naumann EA, Yang CT, et al. Brainwide mapping of neural activity controlling zebrafish exploratory locomotion. eLife. 2016;5:e12741.

12. Friedrich RW, Jacobson GA, Zhu P. Circuit neuroscience in zebrafish. Curr Biol. 2010;20(8):R371-81.

13. Wyart C, Del Bene F. Let there be light: Zebrafish neurobiology and the optogenetic revolution. Rev Neurosci. 2011;22(1):121-30.

14. Brockerhoff SE, Hurley JB, Janssen-Bienhold U, Neuhauss SC, Driever W, Dowling JE. A behavioral screen for isolating zebrafish mutants with visual system defects. Proc Natl Acad Sci U S A. 1995;92(23):10545-9.

15. Burgess HA, Schoch $H$, Granato M. Distinct retinal pathways drive spatial orientation behaviors in zebrafish navigation. Curr Biol. 2010;20(4):381-6.

16. Vitebsky A, Reyes R, Sanderson MJ, Michel WC, Whitlock KE. Isolation and characterization of the laure olfactory behavioral mutant in the zebrafish, danio rerio. Dev Dyn. 2005;234(1):229-42.

17. Gau P, Poon J, Ufret-Vincenty C, Snelson CD, Gordon SE, Raible DW, et al. The zebrafish ortholog of trpv1 is required for heat-induced locomotion. J Neurosci. 2013;33(12):5249-60.

18. Holling C. The functional response of invertebrate predators to prey density. Mem Entomol Soc Can. 1966;98(S48):5-86.

19. Root CM, Ko Kl, Jafari A, Wang JW. Presynaptic facilitation by neuropeptide signaling mediates odor-driven food search. Cell. 2011;145(1):133-44.

20. Filosa A, Barker AJ, Dal Maschio M, Baier H. Feeding state modulates behavioral choice and processing of prey stimuli in the zebrafish tectum. Neuron. 2016:90(3):596-608.

21. Yokogawa T, Hannan MC, Burgess HA. The dorsal raphe modulates sensory responsiveness during arousal in zebrafish. J Neurosci. 2012;32(43):15205-15.

22. Woods IG, Schoppik D, Shi VJ, Zimmerman S, Coleman HA, Greenwood J, et al. Neuropeptidergic signaling partitions arousal behaviors in zebrafish. J Neurosci. 2014;34(9):3142-60

23. Prober DA, Rihel J, Onah AA, Sung RJ, Schier AF. Hypocretin/orexin overexpression induces an insomnia-like phenotype in zebrafish. J Neurosci. 2006:26(51):13400-10.

24. Emran F, Rihel J, Adolph AR, Wong KY, Kraves S, Dowling JE. Off ganglion cells cannot drive the optokinetic reflex in zebrafish. Proc Natl Acad Sci U S A. 2007;104(48):19126-31.

25. Burgess HA, Granato M. Modulation of locomotor activity in larval zebrafish during light adaptation. J Exp Biol. 2007:210(Pt 14):2526-39.

26. Fernandes AM, Fero K, Arrenberg AB, Bergeron SA, Driever W, Burgess HA. Deep brain photoreceptors control light-seeking behavior in zebrafish larvae. Curr Biol. 2012;22(21):2042-7. 
27. Fraenkel GS, Gunn DL. The orientation of animals: Kineses, taxes, and compass reactions. New York: Dover Publications; 1961.

28. Tremblay Y, Roberts AJ, Costa DP. Fractal landscape method: An alternative approach to measuring area-restricted searching behavior. J Exp Biol. 2007; 210(Pt 6):935-45.

29. Budick SA, O'Malley DM. Locomotor repertoire of the larval zebrafish: swimming, turning and prey capture. J Exp Biol. 2000;203(Pt 17):2565-79.

30. Hoffman $\mathrm{G}$. The search behavior of the desert ispod hemilepistus reaumuri as compared with a systematic search. Behav Ecol Sociobiol. 1983;13:93-106.

31. Merkle T, Knaden M, Wehner R. Uncertainty about nest position influences systematic search strategies in desert ants. J Exp Biol. 2006;209(Pt 18):3545-9.

32. Reynolds AM, Smith AD, Menzel R, Greggers U, Reynolds DR, Riley JR. Displaced honey bees perform optimal scale-free search flights. Ecology. 2007:88(8):1955-61.

33. Kareiva P, Odell G. Swarms of predators exhibit "Preytaxis" if individual predators use area-restricted search. Am Nat. 1987;130(2):233-70.

34. Colwill RM, Creton R. Locomotor behaviors in zebrafish (danio rerio) larvae. Behav Process. 2010;86(2):222-9.

35. Chen $X$, Engert F. Navigational strategies underlying phototaxis in larval zebrafish. Front Syst Neurosci. 2014;8:39.

36. Waage J. Foraging for patchily-distrubuted hosts by the parasitoid, nemeritis canescens. J Anim Ecol. 1979;48(2):353-71.

37. Flavell SW, Pokala N, Macosko EZ, Albrecht DR, Larsch J, Bargmann CI. Serotonin and the neuropeptide pdf initiate and extend opposing behavioral states in C. elegans. Cell. 2013;154(5):1023-35.

38. Bailey MR, Jensen G, Taylor K, Mezias C, Williamson C, Silver R, et al. A novel strategy for dissecting goal-directed action and arousal components of motivated behavior with a progressive hold-down task. Behav Neurosci. 2015;129(3):269-80.

39. Salamone JD. Dopaminergic involvement in activational aspects of motivation: effects of haloperidol on schedule-induced activity, feeding, and foraging in rats. Psychobiology. 1988;16:196-206.

40. Bianco $\mathrm{H}$, Kampff AR, Engert F. Prey capture behavior evoked by simple visual stimuli in larval zebrafish. Front Syst Neurosci. 2011:5:101.

41. Schnorr SJ, Steenbergen PJ, Richardson MK, Champagne DL. Measuring thigmotaxis in larval zebrafish. Behav Brain Res. 2012;228(2):367-74.

42. Kokel D, Dunn TW, Ahrens MB, Alshut R, Cheung CY, Saint-Amant L, et al. Identification of nonvisual photomotor response cells in the vertebrate hindbrain. J Neurosci. 2013;33(9):3834-43.

43. Moore HA, Whitmore D. Circadian rhythmicity and light sensitivity of the zebrafish brain. PLoS One. 2014;9(1):e86176.

44. Friedmann D, Hoagland A, Berlin S, Isacoff EY. A spinal opsin controls early neural activity and drives a behavioral light response. Curr Biol. 2015;25(1):69-74.

45. Fischer RM, Fontinha BM, Kirchmaier S, Steger J, Bloch S, Inoue D, et al. Co-expression of val- and tmt-opsins uncovers ancient photosensory interneurons and motorneurons in the vertebrate brain. PLoS Biol. 2013; 11(6):e1001585.

46. Fernandes AM, Beddows E, Filippi A, Driever W. Orthopedia transcription factor otpa and otpb paralogous genes function during dopaminergic and neuroendocrine cell specification in larval zebrafish. PLoS One. 2013;8(9):e75002.

47. Varshney GK, Pei W, LaFave MC, Idol J, Xu L, Gallardo V, et al. Highthroughput gene targeting and phenotyping in zebrafish using crispr/cas9. Genome Res. 2015;25(7):1030-42.

48. Bartumeus F, Peters F, Pueyo S, Marrase C, Catalan J. Helical levy walks: Adjusting searching statistics to resource availability in microzooplankton. Proc Natl Acad Sci U S A. 2003;100(22):12771-5.

49. Reynolds AM, Frye MA. Free-flight odor tracking in drosophila is consistent with an optimal intermittent scale-free search. PLoS One. 2007;2(4):e354.

50. Davies WI, Tamai TK, Zheng L, Fu JK, Rihel J, Foster RG, et al. An extended family of novel vertebrate photopigments is widely expressed and displays a diversity of function. Genome Res. 2016;25(11):1666-79.

51. Vecsei $L$, Schwarzberg $H$, Telegdy $G$. The effect of somatostatin on selfstimulation behavior in atropine- and methysergide-pretreated rats. Eur J Pharmacol. 1983;91(1):89-93.

52. Semenova S, Hoyer D, Geyer MA, Markou A. Somatostatin-28 modulates prepulse inhibition of the acoustic startle response, reward processes and spontaneous locomotor activity in rats. Neuropeptides. 2010:44(5):421-9.

53. Yu K, Garcia da Silva P, Albeanu DF, Li B. Central amygdala somatostatin neurons gate passive and active defensive behaviors. J Neurosci. 2016; 36(24):6488-96.
54. Gahtan E, Tanger P, Baier H. Visual prey capture in larval zebrafish is controlled by identified reticulospinal neurons downstream of the tectum. J Neurosci. 2005;25(40):9294-303.

55. Deeti S, O'Farrell S, Kennedy BN. Early safety assessment of human oculotoxic drugs using the zebrafish visualmotor response. J Pharmacol Toxicol Methods. 2014;69(1):1-8.

56. Emran F, Rihel J, Dowling JE. A behavioral assay to measure responsiveness of zebrafish to changes in light intensities. J Vis Exp. 2008;(20). doi:10.3791/923.

57. Mrosovsky N, Hattar S. Impaired masking responses to light in melanopsinknockout mice. Chronobiol Int. 2003;20(6):989-99.

58. Fernandes AM, Fero K, Driever W, Burgess HA. Enlightening the brain: linking deep brain photoreception with behavior and physiology. Bioessays. 2013;35(9):775-9.

59. LeGates TA, Altimus CM, Wang H, Lee HK, Yang S, Zhao H, et al. Aberrant light directly impairs mood and learning through melanopsin-expressing neurons. Nature. 2012:491(7425):594-8.

60. Decker AR, McNeill MS, Lambert AM, Overton JD, Chen YC, Lorca RA, et al, Abnormal differentiation of dopaminergic neurons in zebrafish trpm7 mutant larvae impairs development of the motor pattern. Dev Biol. 2014; 386(2):428-39.

61. Lambert AM, Bonkowsky JL, Masino MA. The conserved dopaminergic diencephalospinal tract mediates vertebrate locomotor development in zebrafish larvae. J Neurosci. 2012;32(39):13488-500.

62. Low SE, Amburgey K, Horstick E, Linsley J, Sprague SM, Cui WW, et al. Trpm7 is required within zebrafish sensory neurons for the activation of touch-evoked escape behaviors. J Neurosci. 2011:31(32):11633-44.

63. Burgess HA, Granato M. Sensorimotor gating in larval zebrafish. J Neurosci. 2007;27(18):4984-94.

64. Mandelbrot B. The fractal geometry of nature. New York: WH Freeman and Co.; 1982.

65. Coughlin D, Strickler JR, Sanderson B. Swimming and search behaviour in clownfish, amphiprion perideraion, larvae. Anim Behav. 1992;44:427-40.

66. Seuront L, Brewer M, Strickler JR. Quantifying zooplankton swimming behavior: The question of scale. In: Seuront L, Strutton P, editors. Handbook of scaling methods in aquatic ecology. 1st ed. Boca Raton, FL: CRC Press; 2003. p. 333-53.

67. Moreno-Mateos MA, Vejnar CE, Beaudoin JD, Fernandez JP, Mis EK, Khokha MK, et al. Crisprscan: designing highly efficient sgrnas for crispr-cas9 targeting in vivo. Nat Methods. 2015:12(10):982-8.

68. Carrington B, Varshney GK, Burgess SM, Sood R. Crispr-stat: An easy and reliable pcr-based method to evaluate target-specific sgrna activity. Nucleic Acids Res. 2015:43(22):e157.

69. Satou C, Kimura Y, Higashijima S. Generation of multiple classes of v0 neurons in zebrafish spinal cord: progenitor heterogeneity and temporal control of neuronal diversity. J Neurosci. 2012;32(5):1771-83.

70. Horstick EJ, Tabor KM, Jordan DC, Burgess HA. Genetic ablation, sensitization, and isolation of neurons using nitroreductase and tetrodotoxin-insensitive channels. In: Kawakami K, Patton EH, Orger M, editors. Zebrafish: Methods and Protocols, Methods in Molecular Biology, vol. 1451. New York: Springer; 2016. p. 355-66.

\section{Submit your next manuscript to BioMed Central and we will help you at every step:}

- We accept pre-submission inquiries

- Our selector tool helps you to find the most relevant journal

- We provide round the clock customer support

- Convenient online submission

- Thorough peer review

- Inclusion in PubMed and all major indexing services

- Maximum visibility for your research

Submit your manuscript at www.biomedcentral.com/submit 